\title{
Fibrinolytic activity in blood and urine
}

\author{
C. A. C. CHARLTON \\ From the Department of Surgery, Postgraduate Medical School, Hammersmith Hospital, \\ London
}

SYNOPSIS The blood and urinary fibrinolytic activities were simultaneously estimated in 14 'normä' patients under basal conditions on successive days. No direct correlation between the fibrinoly activities in the blood and urine was demonstrated. The origin of urokinase is discussed.

The plasminogen activator found in human urine is known as urokinase. It has been suggested that urokinase represents in part, at least, excreted plasma plasminogen activator (Colgan, Gates, and Miller, 1952). On the other hand, Astrup and Sterndoff (1952) do not believe that this is the case and consider that the urokinase is formed in the urinary tract.

The purpose of this study is to attempt to clarify this problem by simultaneously measuring the fibrinolytic activity in blood and urine, with the subjects at rest and in a basal state.

\section{MATERIALS AND METHODS}

Fourteen patients who were in hospital for investigation of various conditions (which as far as is known were not associated with disturbances of the fibrinolytic system), or had some days previously undergone minor surgical operations, were studied.

The urinary fibrinolytic activity was measured by collecting an overnight 12-hour urine specimen. This was stored at room temperature, since a pilot study had shown that there was no measurable decrease in the fibrinolytic activity of urine stored at room temperature, as compared with that stored at $4^{\circ} \mathrm{C}$., if the subsequent procedures were carried out within a few hours of completing the collection. The volume of the urine was measured. Fresh fibrin plates were prepared by the method of Astrup and Müllertz (1952) with modifications, before each estimation, by making up a $0.5 \%$ solution of bovine fibrinogen (Armour) in normal (physiological) saline, placing $9 \mathrm{ml}$. of this in a petri dish and adding 10 units of thrombin (Leo Laboratories). After approximately half an hour, 3 drops of $0.05 \mathrm{ml}$. of the urine were pipetted on to the fibrin plate at the corners of an equilateral triangle. The plates were incubated in an oven at $37^{\circ} \mathrm{C}$. for 24 hours; at the end of this time, the perpendicular diameters of the lysed areas were measured in millimetres. The products of the diameters $\left(\mathrm{mm} .^{2}\right)$ was calculated, and the mean of the triplicate samples determined.

Received for publication 12 November 1965.
To convert this measurement of $\mathrm{mm}^{2}$ into urokina content (Ploug units) a series of specimens of differeat dilutions of standard urokinase (Leo Laboratories) was prepared. The concentrations varied from 0.5 to 33.0 Ploug units per $\mathrm{ml}$. and the same procedure as previousty described, of adding $0.05 \mathrm{ml}$. of the specimen to the fibain plate and incubating for 24 hours, was carried out. The perpendicular diameters were measured and the prodfft of these $\left(\mathrm{mm} .{ }^{2}\right)$ was plotted as the ordinates against Ge corresponding concentration of urokinase (Ploug urயus per $\mathrm{ml}$.) as the abscissae on double logarithmic grai h paper (Fig. 1). From this graph, the mean of the three estimations obtained with the urine could be conver d into Ploug units of urokinase per millilitre.

Measurements of the patients' urinary fibrinoly activity were determined on at least two, and in so cases four, consecutive days.

The blood fibrinolytic activity was estimated by the dilute blood clot lysis time as described by Fearntery,

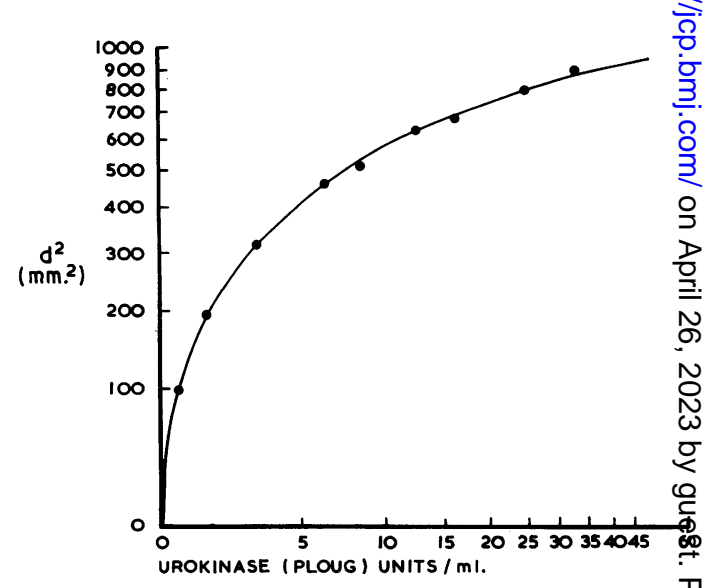

FIG. 1. Plotted on double logarithmic scale, the concentation of urokinase (in Ploug units per $\mathrm{ml}$.) in the stand $\overrightarrow{\text { f }}$ d preparations, as the abscissae against the product of perpendicular diameters $(\mathrm{mm}$.) of the areas lysed, as the ordinates. 
Balmsforth, and Fearnley (1957). At the time corresponding with the end of the 12-hour urine collection, and before the patient had risen from bed or had breakfast, $1.0 \mathrm{ml}$. of blood was obtained by venepuncture (without venous compression); $0.2 \mathrm{ml}$. was immediately pipetted into a test tube containing $1.7 \mathrm{ml}$. of phosphate buffer $(p \mathrm{H} \mathrm{7.4)}$ and $0.1 \mathrm{ml}$. of thrombin (5 units) added, and then shaken and placed in an ice bath. Two such preparations were made for each patient. The tubes were placed in a water bath at $37^{\circ} \mathrm{C}$., and after a few minutes were removed, briskly rotated between thumb and forefinger, so as to free the contracted blood clot from the sides of the test tube, and returned to the bath. The clots were carefully observed until they became irregular on the surface and broke up, the lysis time being the period between introducing the tubes into the bath and the "end point' described.

If lysis of the clot was not complete at the end of 10 hours, little significance could be attached to the further length of time which elapsed, and hence 10 hours was the longest period of time recorded for this measurement. The mean of the two estimations on each occasion was taken as the lysis time, and as in the case of the urine estimations, measurements were made on at least two, and in some cases, four consecutive days for each patient.

\section{RESULTS}

The means and the standard deviations (expressed as a percentage) of fibrinolytic activity in urine and blood are recorded in Table $\mathbf{I}$.

Figure 2 represents the relationship between blood fibrinolytic activity (ordinates) as a function of the urine fibrinolytic activity (abscissae). The blood fibrinolytic activity is expressed as the mean of the reciprocal of the lysis time (Sherry and Alkjaersig, 1957) and the urinary fibrinolytic activity as Ploug units per 12-hour specimen.

As can be seen from Fig. 2, there is no direct

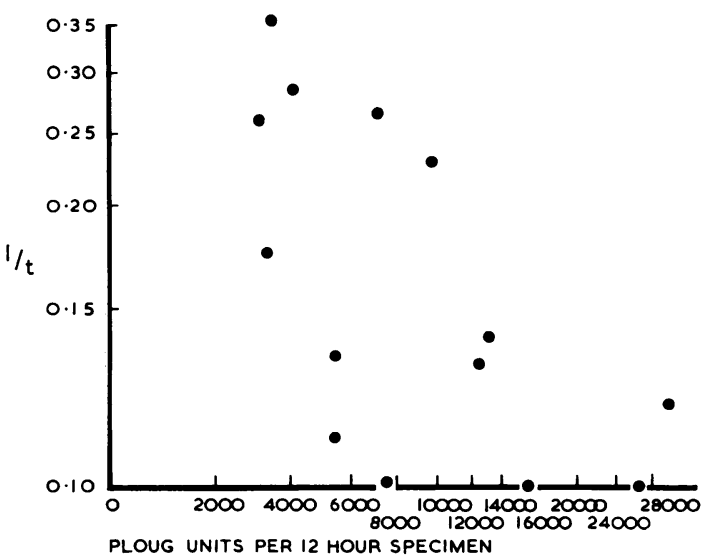

FIG. 2. Plotted on double logarithmic scale, the mean urinary fibrinolytic activity in the 12-hour urine specimens (in Ploug units), as the abscissae against the mean of the reciprocal of the blood lysis time (in hours) for that patient, as the ordinates.

correlation between the fibrinolytic activity of the blood and urine (correlation coefficient equals $-0.005)$. A similar result was obtained if the urinary activity was expressed in Ploug units per litre, and plotted as the abscissae.

\section{DISCUSSION}

The measurement of blood fibrinolytic activity was performed under basal conditions to eliminate the fluctuations known to occur with exercise, mental stress, and ingestion of food (Biggs, Macfarlane, and Pilling, 1947). Under these conditions it was also possible to compare fibrinolytic activity between patients.

TABLE I

FIBRINOLYTIC ACTIVITY OF BLOOD AND URINE

Patient

No.

Urine

Range of Urokinase

Content per 12-Hour

Specimen in Ploug Units
Blood

\begin{tabular}{ll}
\hline Range of Reciprocal & Mean Value and Standard \\
of Lysis Time $(h r)$. & Deviation
\end{tabular}

Deviation
Mean Value and Standard Deviation

$0 \cdot 100-0 \cdot 158$
$0 \cdot 264-0 \cdot 316$
$0 \cdot 304-0 \cdot 461$
$0 \cdot 159-0 \cdot 218$
$0 \cdot 100-0 \cdot 245$
$0 \cdot 100-0 \cdot 103$
$0 \cdot 125-0 \cdot 156$
$0 \cdot 218-0 \cdot 317$
$0 \cdot 179-0 \cdot 317$
$0 \cdot 100-0 \cdot 142$
$0 \cdot 100-0 \cdot 100$
$0 \cdot 100-0 \cdot 218$
$0 \cdot 100-0 \cdot 100$
$0 \cdot 218-0 \cdot 304$

$0 \cdot 116 \pm 24 \%$

$0 \cdot 286 \pm 9 \%$

$0.364 \pm 19 \%$

$0.178 \pm 16 \%$

$0.157 \pm 42 \%$

$0.102 \pm 2 \%$

$0.141 \pm 16 \%$

$0.267 \pm 26 \%$

$0.248 \pm 40 \%$

$0.140 \pm 28 \%$

$0.100 \pm 0 \%$

$0.139 \pm 49 \%$

$0.100 \pm 0 \%$

$0 \cdot 271 \pm 17 \%$ 
Although it has been suggested that blood fibrinolytic activity is fairly constant under basal conditions (Fearnley, 1964), the results of this study (Table I) showed a considerable day-to-day fluctuation.

This could, in part, be attributed to technical errors, but the same procedure was carried out by me throughout, and the difference between duplicates did not exceed $10 \%$. A true variation in basal fibrinolytic activity due to fluctuation in plasminogen activator level is unlikely; however, the fluctuation noted could be due to unrecognized stimuli occurring during the apparently basal conditions.

The urinary fibrinolytic activity also showed considerable day-to-day variation (Table I) but the agreement between the triplicate assays was of a high order.

If the urinary fibrinolytic activity represents filtered plasma activator activity (plasminogen activator) it might be expected that fluctuations in these two systems would occur in parallel. However, this was not the case, and in a number of patients, even marked fluctuations in plasma fibrinolytic activity were not reflected in the urinary fibrinolytic activity.

The results suggest that there is no correlation between the levels of plasminogen activator and urinary activator (urokinase) when the activities are measured under basal conditions. Superficially, this appears to contradict the findings of von Kaulla and Swan (1958) who investigated fibrinolytic activity during open heart surgery and found a correlation between increased blood and urinary fibrinoly activity. Similar findings were also noted in dogs Colgan et al. (1952), following total body irradiation.

However, in both of the above reports, the plasrifa fibrinolytic activity was increased well beyo physiological levels and these studies are not reaty comparable with the one performed here.

It is possible that urinary fibrinolytic activi represents the sum of locally produced and filtered activator activities, and that the relative contributions of these two varies with different conditions..

Thus, under basal conditions with a low level of circulating plasminogen activator, urokinase consiss mainly of locally produced activator. However, whe्en plasminogen activator levels are markedly increased, a significant amount is filtered into the urine ah contributes to the overall urinary fibrinolytic activity.

I thank Professor R. Shackman for permission $\Phi_{0}$ investigate patients under his care, and for his encouragement, and Dr. J. Hirsh for his most helpful criticism?

\section{REFERENCES}

Astrup, T., and Müllertz, S. (1952). Arch. Biochem., 40, 346. - and Sterndoff, I. (1952). Proc. Soc. exp. Biol. (N.Y.), 81, 675 Biggs, R., Macfarlane, R. G., and Pilling, J. (1947). Lancet, 1, 40. Colgan, J., Gates, E., and Miller, L. L. (1952). J. exp. Med., 95, 5 ş1. Fearnley, G. R. (1964). Brit. med. Bull., 20, 185.

-, Balmsforth, G., and Fearnley, E. (1957). Clin. Sci., 16, 645. O von Kaulla, K. N., and Swan, H. (1958). J. thorac. Surg., 36, 5190 Sherry, S., and Alkjaersig, N. (1957). Thrombos. Diathes. haemorgh (Stuttg.), 1, 264. 